\title{
Constraint Satisfaction Problems of Bounded Width
}

\author{
Libor Barto and Marcin Kozik
}

April 2, 2009

\begin{abstract}
We provide a full characterization of applicability of The Local Consistency Checking algorithm to solving the non-uniform Constraint Satisfaction Problems. This settles the conjecture of Larose and Zádori.
\end{abstract}

\section{Introduction}

The Constraint Satisfaction Problem (CSP) is one of few problems central to the development of theoretical computer science. The computational complexity and approximability problems for CSP attracted attention of researchers for a long time. One of the most significant motivations for studying CSP is it's applicability - it provides a common framework for many combinatorial problems in artificial intelligence and applied computer science.

An instance of CSP consists of variables and constraints and the aim is to determine whether variables can be evaluated in such a way that all the constraints are satisfied. One of the most studied forms of CSP are non-uniform CSPs, where constraints are taken from a fixed finite set called the template. Examples of such problems include $k$-SAT, Graph $H$-Coloring, System of EQUATIONS and many others. The most famous open question in this field is the Dichotomy Conjecture of Feder and Vardi [13], postulating that every non-uniform CSP is solvable in polynomial time or NP-complete.

The Dichotomy Conjecture has proved to be a challenging question and advances using standard methods were slow. A breakthrough in the development occurred when Jeavons, Cohen and Gyssens [14] announced an algebraic approach to the problem. Their work, refined later by Bulatov, Jeavons and Krokhin $[10,6]$, showed that the complexity of a non-uniform CSP is fully determined by a set of operations - the polymorphisms of the template. The algebraic approach allowed to conjecture the precise boundary between NP-complete and tractable problems [10] and led to results unreachable before, e.g. [7], [9], [2, 3].

All known non-uniform tractable CSPs are solvable by a combination of two algorithms, or rather algorithmic principles. One of them is based on the "few subpowers property" [5, 4] and its applicability to solve CSPs is already fully understood [4]. The other one, the Local Consistency Checking, is definitely the widest known and the most natural algorithm for solving CSP. However, it was an open problem to determine for which templates the corresponding CSP can be solved by this algorithm (which templates have bounded width). This problem was considered to be crucial before attacking the Dichotomy Conjecture. A plausible conjecture characterizing such problems was proposed by Larose and Zádori in [16]. They proved that if a template has bounded width, then the algebra associated with it (where operations of the algebra are polymorphisms of the template) lies in a congruence meet semi-distributive variety, and conjectured the converse. This paper confirms the characterization they postulated. 
Our proof of this fact is a mixture of the following ingredients: a characterization of congruence meet semi-distributivity by Maróti and McKenzie [17]; techniques developed in [1] for proving a special case of the Bounded Width Conjecture, which are here substantialized in the notion of Prague strategy and Theorem 4.12; and new algebraic results, which are stated as Theorem 4.10 and Theorem 4.11.

\section{Preliminaries}

It is well known (see e.g. [13]) that the Constraint Satisfaction Problem can be formulated as a homomorphism problem for relational structures. To prove the claimed result we work with relational structures enriched by their algebraic counterparts. In this section we introduce basic notions concerning relational structures, algebras and polymorphisms.

\subsection{Relational structures}

An $n$-ary relation on a set $A$ is a subset of $A^{n}$. A relational structure is a tuple $\mathbb{A}=\left(A, R_{0}, R_{1}, \ldots\right)$, where $A$ is a set (the universe of $\mathbb{A}$ ) and $R_{0}, R_{1} \ldots$, are relations on $A$. All relational structures in this paper are assumed to be finite and with a finite number of relations.

Relational structures $\mathbb{A}=\left(A, R_{0}, R_{1}, \ldots\right), \mathbb{B}=\left(B, S_{0}, S_{1} \ldots\right)$ have the same type, if they have the same number of relations and the relation $R_{i}$ has the same arity (denoted by $\operatorname{ar}_{i}$ ) as $S_{i}$ for every $i$. In such a situation, a mapping $f: A \rightarrow B$ is called a homomorphism from $\mathbb{A}$ to $\mathbb{B}$ if it preserves all the relations, that is for every $i$ and every $\left(a_{1}, \ldots, a_{\mathrm{ar}_{i}}\right) \in R_{i}$, we have $\left(f\left(a_{1}\right), \ldots, f\left(a_{\mathrm{ar}_{i}}\right)\right) \in S_{i}$. If there exists a homomorphism from $\mathbb{A}$ to $\mathbb{B}$ we say that $\mathbb{A}$ is homomorphic to $\mathbb{B}$. If every homomorphism $\mathbb{A} \rightarrow \mathbb{A}$ is bijective we call $\mathbb{A}$ a core.

\section{$2.2 \quad$ Algebras}

An $n$-ary operation on the set $A$ is a mapping $A^{n} \rightarrow A$. Such an operation $t$ is called idempotent, if $t(a, a, \ldots, a)=a$ for all $a \in A$.

An algebra is a tuple $\mathbf{A}=\left(A, t_{0}, t_{1}, \ldots\right)$, where $A$ is a nonempty set (called a universe) and $t_{0}, t_{1}, \ldots$ are operations on $A$. Similarly as with relational structures, algebras $\mathbf{A}, \mathbf{B}$ are of the same type if they have the same number of operations and corresponding operations have equal arities. By abuse of notation we sometimes denote operations of two algebras of the same type by the same symbols. When there is a danger of confusion, we emphasize the appropriate algebra in superscript, e.g. $\mathbf{A}=\left(A, t_{0}^{\mathbf{A}}, \ldots\right)$.

A term operation of an algebra is any operation that can be obtained as a composition using the operations of the algebra together with all the projections. The set of all term operation of $\mathbf{A}$ is denoted by Clo $\mathbf{A}$.

A mapping $f: A \rightarrow B$ is a homomorphism of algebras, if it preserves all the operations, that is $f\left(t_{i}\left(a_{1}, \ldots, a_{\mathrm{ar}_{i}}\right)\right)=t_{i}\left(f\left(a_{1}\right), f\left(a_{2}\right), \ldots, f\left(a_{\mathrm{ar}_{i}}\right)\right)$ for any $i$ and any $a_{1}, a_{2}, \cdots \in A$. A bijective homomorphism is an isomorphism.

A set $B \subseteq A$ is a subuniverse of $\mathbf{A}$ if, for every $i$, the operation $t_{i}$ restricted to $B^{\text {ar }_{i}}$ has all the results in $B$. For a nonempty subuniverse $B$ of an algebra $\mathbf{A}$ the algebra $\mathbf{B}=\left(B, t_{0}^{\prime}, \ldots\right)$ (where $t_{i}^{\prime}$ is a restriction of $t_{i}$ to $\left.B^{\mathrm{ar}_{i}}\right)$ is a subalgebra of $\mathbf{A}$. A set $C \subseteq A$ generates a subuniverse $B$ in an algebra $\mathbf{A}$ if $B$ is the smallest subuniverse containing $C$ - such a subuniverse always exists and can be obtained by applying all the term operations of the algebra $\mathbf{A}$ to all the choices of arguments coming from $C$. 
Given algebras $\mathbf{A}, \mathbf{B}$ of the same type, a product $\mathbf{A} \times \mathbf{B}$ of $\mathbf{A}$ and $\mathbf{B}$ is the algebra with the same type as $\mathbf{A}$ and $\mathbf{B}$ with universe $A \times B$ and operations computed coordinatewise. An algebra $\mathbf{C}$ is a subdirect product of $\mathbf{A}$ and $\mathbf{B}$ if it is a subalgebra of $\mathbf{A} \times \mathbf{B}$ such that the projections of $C$ to $A$ and $B$ are full. For a set $H$, an $H$-power $\mathbf{A}^{H}$ of an algebra $\mathbf{A}$ has a universe $A^{H}$ (the set of all the mappings from $H$ to $A$ ) and the operations are again computed coordinatewise (the algebra $\mathbf{A}^{H}$ is naturally isomorphic to $\mathbf{A} \times \cdots \times \mathbf{A}$, where the product is taken $|H|$-times.)

An equivalence relation $\theta$ on $A$ is called a congruence of an algebra $\mathbf{A}$ if $\theta$ is a subalgebra of $\mathbf{A} \times \mathbf{A}$. Given a congruence $\theta$ on $A$, we can form the factor algebra $\mathbf{A} / \theta$ of the same type as $\mathbf{A}$, whose elements are the equivalence classes of $\mathbf{A}$ and the operations are defined so that the natural projection mapping is a homomorphism $\mathbf{A} \rightarrow \mathbf{A} / \theta$. If $\mathbf{A}$ is idempotent, then $\theta$-classes are subuniverses of $\mathbf{A}$. An algebra $\mathbf{A}$ is simple, if it has the trivial congruences only (i.e. the diagonal and the full congruence). If $\theta$ is a maximal congruence of $\mathbf{A}$, then $\mathbf{A} / \theta$ is simple.

The set of all congruences of an algebra $\mathbf{A}$ with the inclusion relation forms a lattice, that is a partially ordered set such that all two-element subsets $\{x, y\}$ have supremum $x \vee y$ and infimum $x \wedge y$.

A variety is a class of algebras of the same type closed under forming isomorphic algebras, subalgebras, products, and factor algebras.

\subsection{Polymorphisms}

An operation $t: A^{m} \rightarrow A$ is compatible with a relation $R \subseteq A^{n}$ if

$$
\left(t\left(a_{11}, \ldots, a_{1 m}\right), \ldots, t\left(a_{n 1}, \ldots, a_{n m}\right)\right) \in R
$$

whenever $\left(a_{11}, \ldots, a_{n 1}\right), \ldots,\left(a_{1 m}, \ldots, a_{n m}\right) \in R$. An operation $t: A^{m} \rightarrow A$ is a polymorphism of a relational structure $\mathbb{A}$ if it is compatible with all the relations of $\mathbb{A}$.

To every core relational structure $\mathbb{A}$ we associate an algebra $\mathbf{A}$ which operations are all the idempotent polymorphisms of $\mathbb{A}$ (in an arbitrarily chosen order). It is easy to see that every projection is a polymorphism of any relational structure and that the set of all polymorphisms of a relational structure is closed under forming compositions. Therefore every term operation of such constructed $\mathbf{A}$ is an operation of $\mathbf{A}$.

\section{CSP and the conjecture of Larose and Zádori}

Let $\mathbb{A}$ be a relational structure (with a finite universe and a finite number of relations, each of a finite arity). The Constraint Satisfaction Problem with template $\mathbb{A}, \operatorname{CSP}(\mathbb{A})$, is the following decision problem:

INPUT: A relational structure $\mathbb{X}$ of the same type as $\mathbb{A}$.

QUESTION: Does there exist a homomorphism $\mathbb{X} \rightarrow \mathbb{A}$ ?

The Dichotomy Conjecture of Feder and Vardi [13] states that each problem $\operatorname{CSP}(\mathbb{A})$ is tractable or NP-complete (we note that the conjecture trivializes unless $\mathrm{P} \neq \mathrm{NP}$ ).

It is easy to see that, for every $\mathbb{A}, \operatorname{CSP}(\mathbb{A})$ is the same as a $\operatorname{CSP}\left(\mathbb{A}^{\prime}\right)$ for a certain core $\mathbb{A}^{\prime}$ (called the core of $\mathbb{A}$ ) - this allows us to restrict the reasoning to relational structures which are cores.

The algebra $\mathbf{A}$ associated with $\mathbb{A}$ (in a sense of subsection 2.3) plays a key role in the algebraic approach to CSP: it is known $[14,10,6]$ that $\mathbf{A}$ determines the complexity of $\operatorname{CSP}(\mathbb{A})$. Thus a task of classifying the computational complexities of various constraint satisfaction problems reduces to a classification of algebras that arise as their counterparts. Here operations of the algebra of a particular shape play a crucial role. 
Definition 3.1. An $n$-ary $(n \geq 2)$ operation $t$ on $A$ is called weak near-unanimity (WNU), if it is idempotent and, for all $a, b \in A$,

$$
t(a, a, \ldots, a, b)=t(a, a, \ldots, a, b, a)=\cdots=t(b, a, a, \ldots, a) .
$$

By a combination of results from $[10,6,17]$ it follows that if $\mathbf{A}$ has no weak near-unanimity operation, then $\operatorname{CSP}(\mathbb{A})$ is NP-complete. The Algebraic Dichotomy Conjecture states that the converse also holds:

Conjecture 3.2 (The Algebraic Dichotomy Conjecture). Let $\mathbb{A}$ be a core relational structure. The $\operatorname{CSP}(\mathbb{A})$ is tractable, if $\mathbf{A}$ has a WNU operation and it is NP-complete otherwise.

Thus the algebraic dichotomy conjecture is a strengthening of the conjecture of Feder and Vardi.

\subsection{Problems of bounded width}

The definition of a CSP of bounded width can be introduced in a number of equivalent ways (using duality, infinitary logic, pebble games, Datalog programs, strategies), see [16, 11]. We will use the approach via strategies.

Definition 3.3. Let $\mathbb{X}, \mathbb{A}$ be relational structures of the same type and $k \leq l$ be natural numbers. $A$ set $\mathcal{F}$ of partial homomorphisms from $\mathbb{X}$ to $\mathbb{A}$ is called a $(k, l)$-strategy for $(\mathbb{X}, \mathbb{A})$, if it satisfies the following:

$(S 1)|\operatorname{dom}(f)| \leq l$, for any $f \in \mathcal{F}$.

$(S 2)$ For any $f \in \mathcal{F}$ and any $K \subseteq \operatorname{dom}(f)$ the function $f_{\mid K}$ belongs to $\mathcal{F}$.

(S3) For any $K \subseteq L \subseteq A$ with $|K| \leq k,|L| \leq l$, and $f \in \mathcal{F}$ with $\operatorname{dom}(f)=K$, there exists $g \in \mathcal{F}$ such that $\operatorname{dom}(g)=L$ and $g_{\mid K}=f$.

For $K \subseteq X$ with $|K| \leq l$ the set of all partial homomorphisms from $\mathcal{F}$ with domain $K$ will be denoted by $\mathcal{F}_{K}$, that is $\mathcal{F}_{K}=\mathcal{F} \cap X^{K}$.

A standard procedure [13] called $(k, l)$-Consistency Checking, finds the greatest (with respect to inclusion) $(k, l)$-strategy $\mathcal{F}$ for $(\mathbb{X}, \mathbb{A})$. The algorithm starts by throwing initially in $\mathcal{F}$ all partial homomorphisms (from $\mathbb{X}$ to $\mathbb{A}$ ) with domain of size less or equal to $l$. Then we repeatedly remove from $\mathcal{F}$ all the mappings falsifying condition $(S 2)$ or $(S 3)$ until all the conditions are satisfied. It is not difficult to see that this algorithm runs in polynomial time with respect to $|X|$.

Moreover note that for any choice of $K$, any $f_{1}, \ldots, f_{m} \in \mathcal{F}_{K}$ and any $m$-ary polymorphism $t$ of $\mathbb{A}$ the function $t\left(f_{1}, \ldots, f_{m}\right)$ is a partial homomorphism from $\mathbb{X}$ to $\mathbb{A}$. Using this fact it is easy to prove that every $(k, l)$-strategy for $(\mathbb{X}, \mathbb{A})$ can be enlarged to a new $(k, l)$-strategy for $(\mathbb{X}, \mathbb{A})$ where each the $\mathcal{F}_{K}$ is a subalgebra of $\mathbf{A}^{K}$. Since the $(k, l)$ strategy produced for $(\mathbb{X}, \mathbb{A})$ by the local consistency checking was the greatest possible it has to satisfy the same condition. Thus polymorphisms of a relational structure appear naturally in the strategies for which the structure is taken as a target.

Observe that, for any homomorphism $f: \mathbb{X} \rightarrow \mathbb{A}$ and any $K \subseteq X$ with $|K| \leq l$, the partial homomorphism $f_{\mid K}$ belongs to the strategy returned by the $(k, l)$-consistency algorithm. Therefore if the algorithm returns $\mathcal{F}=\emptyset$ then there is certainly no homomorphism from $\mathbb{X}$ to $\mathbb{A}$. The structure $\mathbb{A}$ is of width $(k, l)$ if the converse is also true:

Definition 3.4. A relational structure $\mathbb{A}$ has width $(k, l)$ if for every relational structure $\mathbb{X}$, if there exists a nonempty $(k, l)$-strategy for $(\mathbb{X}, \mathbb{A})$ then $\mathbb{X}$ is homomorphic to $\mathbb{A}$. Moreover $\mathbb{A}$ is said to be of width $k$, if it has width $(k, l)$ for some $l$, and to be of bounded width if it has width $k$ for some $k$. 
In other words, a relational structure $\mathbb{A}$ has bounded width, if there exist $k$ and $l$ such that we can use the $(k, l)$-Consistency Checking algorithm to solve $\operatorname{CSP}(\mathbb{A})$. As noted above, this algorithm works in polynomial time, thus if $\mathbb{A}$ has bounded width then $\operatorname{CSP}(\mathbb{A})$ is tractable.

\subsection{Congruence meet semi-distributive algebras}

A particular lattice identity has a close connection with problems of bounded width:

Definition 3.5. A lattice $\mathbb{L}$ is meet semi-distributive, if $a \wedge b=a \wedge c$ implies $a \wedge(b \vee c)=a \wedge b$ for all $a, b, c \in L$.

A variety $\mathcal{V}$ is called congruence meet semi-distributive if all the algebras in $\mathcal{V}$ have meet semidistributive congruence lattices. Maróti and McKenzie [17] proved that a finite algebra A lies in a congruence meet semi-distributive variety, if and only if $\mathbf{A}$ has WNU term operations of all but finitely many arities. We will call such algebras $S D(\wedge)$ algebras.

\subsection{The conjecture of Larose and Zádori}

Larose and Zádori [16] proved that if a core $\mathbb{A}$ has bounded width, then $\mathbf{A}$ is an $S D(\wedge)$ algebra, and conjectured the converse:

Conjecture 3.6 (The Bounded Width Conjecture). A core relational structure $\mathbb{A}$ has bounded width if and only if $\mathbf{A}$ is an $S D(\wedge)$ algebra, i.e. A has $W N U$ operations of all but finitely many arities.

This conjecture was verified in several special cases [13], [8], [15], [12], [1] and the main result of this paper confirms it in full generality:

Theorem 3.7. Let $\mathbb{A}$ be a relational structure such that $\mathbf{A}$ is an $S D(\wedge)$ algebra. Then $\mathbb{A}$ has width $\left(2\left\lceil\frac{p}{2}\right\rceil, 3\left\lceil\frac{p}{2}\right\rceil\right)$, where $p$ is the maximal arity of a relation in $\mathbb{A}$.

\section{A proof of Theorem 3.7}

The proof of Theorem 3.7 is split into a number of subsections with some of the proofs moved to the appendix. In subsection 4.1 we introduce a notion of a Prague strategy and show a reduction of Theorem 3.7 to Theorem 4.5. In subsection 4.2 we present algebraic facts (proved in the appendix) required in the reminder of the proof. Finally, in subsections 4.3 and 4.4, we present a proof of Theorem 4.5 split into two, mutually exclusive, cases.

\subsection{Reduction to Prague strategies}

In order to prove Theorem 3.7 we work with a relational structure $\mathbb{A}$ and number $p$ as in the statement. Moreover we fix an arbitrary relational structure $\mathbb{X}$ such that there exists a non-empty $\left(2\left\lceil\frac{p}{2}\right\rceil, 3\left\lceil\frac{p}{2}\right\rceil\right)$ strategy for the pair $(\mathbb{X}, \mathbb{A})$. Note that if we found a function $f$ from $\mathbb{X}$ to $\mathbb{A}$ such that $f_{\mid K}$ is in the strategy for all $K \subseteq X$ with at most $p$ elements then $f$ would be a homomorphism from $\mathbb{X}$ to $\mathbb{A}$. Existence of such an $f$ is provided by Theorem 4.5.

In the following few definitions we introduce a notion of a Prague strategy and show a reduction of Theorem 3.7 to Theorem 4.5.

Definition 4.1. $A(1,2)$-system $\mathcal{B}$ (of size $n$ ) is a collection of finite nonempty sets $\mathcal{B}=\left\{B_{i}, B_{i, j}\right.$ : $0 \leq i, j<n\}$ such that for any $i, j<n$ 
(B1) $B_{i, j} \subseteq B_{i} \times B_{j}, B_{i, i}=\left\{(a, a): a \in B_{i}\right\}, B_{i, j}=\left\{(a, b):(b, a) \in B_{j, i}\right\}$, and

(B2) $B_{i, j}$ is subdirect, i.e. the projections of $B_{i, j}$ to both coordinates are full.

$A$ solution of a $(1,2)$-system $\mathcal{B}$ is a tuple $\left(b_{0}, \ldots, b_{n-1}\right)$ such that $\left(b_{i}, b_{j}\right) \in B_{i, j}$ for any $i, j<n$.

A $(1,2)$-system (of size $n$ ) can be viewed as a $n$-sorted graph with $B_{i}$ 's as sorts of vertices and $B_{i, j}$ 's as sorts of edges. A multisorted version of a connectivity requires the following definition.

Definition 4.2. Let $\mathcal{B}$ be a $(1,2)$-system. $A$ pattern in $\mathcal{B}$ is a finite sequence of natural numbers smaller than $n$. For patterns $w, v$ we write $w v$ for concatenation of patterns $w$ and $v$ and $w^{k}$ for a $k$-ary concatenation of $w$ with itself. We write $w^{-1}$ for a pattern $w$ with reversed order and set $w^{-k}=\left(w^{-1}\right)^{k}$ for any $k$.

While working with patterns we use $\llbracket w \rrbracket$ to denote a set of all numbers that appear in $w$. Moreover we say that a pattern $w$ is based at $i$ if the first and the last number of $w$ are equal to $i$. Using patterns we can define connectivity in $(1,2)$-systems.

Definition 4.3. Let $\mathcal{B}$ be a $(1,2)$-system, then

- A sequence $a_{0}, \ldots a_{l}$ is called a realization of a pattern $w=\left(w_{0}, \ldots, w_{l}\right)$ in $\mathcal{B}$, if $a_{i} \in B_{w_{i}}$ for all $i \leq l$ and $\left(a_{i}, a_{i+1}\right) \in B_{w_{i}, w_{i+1}}$ for all $i<l$.

- We say that two elements $a \in B_{i}, b \in B_{j}$ are connected via a pattern $w=(i, \ldots, j)$ (in $\left.\mathcal{B}\right)$, and write $a \stackrel{w}{\longrightarrow} b$, if there exists a realization $a=a_{0}, a_{1}, \ldots, a_{l}=b$ of the pattern $w$.

Moreover we say that two elements $a \in B_{i}$ and $b \in B_{j}$ can be connected in a set of natural numbers $K$ if they are connected via a pattern $w=(i, \ldots, j)$ such that $\llbracket w \rrbracket \subseteq K$.

Definition 4.4. $A(1,2)$-system $\mathcal{B}$ is a Prague strategy, if

(B4) for every number $i<n$ and every pattern $w$ based at $i$ in $\mathcal{B}$ if $a, b \in B_{i}$ are connected in $\llbracket w \rrbracket$, then there exists $k>0$ such that $a \stackrel{w^{k}}{\longrightarrow} b$.

Moreover we say that a Prague strategy $\mathcal{B}$ is compatible with $\mathbf{A}$ if each $B_{i}$ is a subuniverse of $\mathbf{A}$ and each $B_{i, j}$ is a subuniverse of $\mathbf{A} \times \mathbf{A}$.

The following theorem is the main focus of the paper. A reduction of Theorem 3.7 to Theorem 4.5 is presented below with a gap, which we fill up in the appendix.

Theorem 4.5. Every Prague strategy compatible with an $S D(\wedge)$ algebra has a solution.

Sketch of a proof for Theorem 3.7. Let $q=\left\lceil\frac{p}{2}\right\rceil$ and let $\mathcal{F}$ be a maximal (wrt. inclusion) $(2 q, 3 q)$ strategy for $(\mathbb{X}, \mathbb{A})$. We define a $(1,2)$-system indexed by $q$-element subsets of $X$ (instead of natural numbers) by putting $B_{I}=\mathcal{F}_{I}$ and $B_{I, J}=\left\{(f, g) \in B_{I} \times B_{J}: \exists h \in \mathcal{F}_{I \cup J} h_{\mid I}=f, h_{\mid J}=g\right\}$. Then $\mathcal{B}=\left\{B_{I}, B_{I, J}: I, J \subseteq X,|I|=|J|=q\right\}$ is, by remarks after Definition 3.3, compatible with the $S D(\wedge)$ algebra $\mathbf{A}^{q}$ (although formally $B_{I}$ is not a subuniverse of $\mathbf{A}^{q}$ and rather a subuniverse of $\mathbf{A}^{I}$ the problem is a technicality) In the appendix we show that $\mathcal{B}$ is a Prague strategy. A solution of $\mathcal{B}$ provided by Theorem 4.5 gives us a homomorphism $\mathbb{X} \rightarrow \mathbb{A}$.

The key part of the proof of Theorem 4.5 deals with finding subsystems of given (1,2)-system. If $\mathcal{B}$ is a $(1,2)$-system (consisting of $B_{i}$ 's and $B_{i, j}$ 's) and $\mathcal{C}$ is a $(1,2)$-system consisting of $C_{i}$ 's and $C_{i, j}$ 's such that $C_{i} \subseteq B_{i}$ and $C_{i, j}=B_{i, j} \cap\left(C_{i} \times C_{j}\right)$ for every $i, j<n$ then we call $\mathcal{C}$ a subsystem of $\mathcal{B}$. If at least one of the inclusions is strict we call it a proper subsystem. We need two definitions of substrategies. 
Definition 4.6. Let $\mathcal{B}$ be a Prague strategy and let $\mathcal{C}$ be a subsystem of $\mathcal{B}$ (treated as a $(1,2)$ system). If $\mathcal{C}$ is a Prague strategy then it is a substrategy of $\mathcal{B}$. Moreover if

$(B 4 S)$ for every number $i<n$ and every pattern $w$ based at $i$ in $\mathcal{B}$ if $a, b \in C_{i}$ and $a, b$ are connected in $\llbracket w$ in the strategy $\mathcal{B}$, then there exists $k>0$ such that $a \stackrel{w^{k}}{\longrightarrow} b$ in $\mathcal{C}$

then $\mathcal{C}$ is a strong substrategy of $\mathcal{B}$.

We write $\mathcal{B}_{w}(K)$ to denote the set of all the elements reachable (in a $(1,2)$-system $\left.\mathcal{B}\right)$ from $K \subseteq$ $B_{i}$ via a pattern $w$ (beginning with $i$ ). Moreover we sometimes write $\mathcal{B}_{i, j}(K)$ for $\mathcal{B}_{(i, j)}(K)$ (where $w$ is taken to be $(i, j))$. Finally we introduce two new conditions equivalent (for a $(1,2)$-system) to condition $(B 4)$ :

Lemma 4.7. Let $\mathcal{B}$ be $(1,2)$-system. TFAE:

$(B 4) \mathcal{B}$ is a Prague strategy;

(B5) for every number $i<n$ and every pattern $w$ based at $i$ in $\mathcal{B}$ if $a, b \in B_{i}$ are connected in $\llbracket w \rrbracket$, then there exists a natural number $m$ such that, for all $k>m, a \stackrel{w^{k}}{\longrightarrow} b$;

(B6) for every number $i<n$ and every pattern $w$ based at $i$ in $\mathcal{B}$ if $K_{0} \subseteq B_{i}$ and $\mathcal{B}_{w}\left(K_{0}\right)=K_{0}$ then for any initial segment $w^{\prime}$ of $w$ ending with $j \mathcal{B}_{w^{\prime}}\left(K_{0}\right)=\mathcal{B}_{i, j}\left(K_{0}\right)$.

\subsection{Algebraic results}

For a given algebra $\mathbf{A}$ we distinguish some of its subuniverses. We say that a non-empty subuniverse $K$ absorbs an algebra $\mathbf{A}$ wrt. term $t$, if the term operation $t$ of $\mathbf{A}$ satisfies

$$
t(K, K, \ldots, K, A) \cup t(K, K, \ldots, K, A, K) \cup \cdots \cup t(A, K, K, \ldots, K) \subseteq K .
$$

Moreover we say that $K$ absorbs $\mathbf{A}$ (or $K$ is an absorbing subuniverse of $\mathbf{A}$ ), if there exists an operation $t$ such that $K$ absorbs $\mathbf{A}$ wrt. $t$.

Definition 4.8. Let $R \subseteq C \times D$. We say that $c, c^{\prime} \in C$ are linked in $R$, if there exist a natural number $i, c=c_{0}, c_{1}, \ldots, c_{i}=c^{\prime} \in C$ and $d_{1}, \ldots, d_{i} \in D$ such that for all $0 \leq j<i$ we have $\left(c_{j}, d_{j+1}\right) \in R$ and $\left(c_{j+1}, d_{j+1}\right) \in R$.

We say that $R$ is fully linked, if every two elements of $C$ are linked in $R$ and we say that $R$ is left separated, if $(c, d),\left(c^{\prime}, d\right) \in R$ implies $c=c^{\prime}$ for all $c, c^{\prime} \in C$ and $d \in D$.

These definitions allow us to introduce a simple lemma and two more involved theorems with proofs in the appendix.

Lemma 4.9. Let $\mathbf{R}$ be a subdirect product of $\mathbf{C}$ and $\mathbf{D}$. If $\mathbf{C}$ is simple and $R$ is not left separated, then $R$ is fully linked.

Theorem 4.10. Let $\mathbf{A}$ be a finite $S D(\wedge)$ algebra. Let $\mathcal{R}$ be a family of subsets of $A$ satisfying the following conditions

- $A \in \mathcal{R}$

- If $t \in$ Clo $\mathbf{A}$ is a WNU operation, $X \in \mathcal{R}$ and $x \in X$, then $\{t(x, x, \ldots, x, y): y \in X\} \in \mathcal{R}$

Then $\mathcal{R}$ contains a one-element set.

Theorem 4.11. Let $\mathbf{A}$ be a finite $S D(\wedge)$ algebra. Let $\mathbf{C}, \mathbf{D} \leq \mathbf{A}$ and $\mathbf{R} \leq \mathbf{C} \times \mathbf{D}$ be fully linked and subdirect. Then either $R=C \times D$ or there exist a proper absorbing set in $\mathbf{C}$ or $\mathbf{D}$. 


\subsection{The absorbing case}

The following theorem was essentially discovered already in [1].

Theorem 4.12. Let $\mathcal{B}$ be a Prague strategy of size $n$ compatible with an algebra $\mathbf{A}$. If, for some $i_{0}<n$ and $K_{0}$ a proper subuniverse of $B_{i_{0}}, K_{0}$ absorbs $\mathbf{B}_{i_{0}}$ then there exists a proper strong substrategy of $\mathcal{B}$ compatible with $\mathbf{A}$.

Proof. Let $t \in$ Clo $\mathbf{A}$ be such that $K_{0}$ absorbs $\mathbf{B}_{i_{0}}$ wrt. $t^{\mathbf{B}_{i_{0}}}$ and let $\mathcal{K}=\left\{(D, i): i<n, D \varsubsetneqq B_{i}\right\}$. We define a preorder $\leq$ on $\mathcal{K}$ putting

$$
(D, i) \leq(E, j) \text { iff there exists a pattern } w=(i, \ldots, j) \text { such that } \mathcal{B}_{w}(D)=E .
$$

Claim. (1) If $(D, i) \leq(E, j)$ and $D$ absorbs $\mathbf{B}_{i}$ wrt. $t^{\mathbf{B}_{i}}$, then $E$ absorbs $\mathbf{B}_{j}$ wrt. $t^{\mathbf{B}_{j}}$.

Proof. Let $w$ be a pattern such that $\mathcal{B}_{w}(D)=E$. Let $q$ be the arity of $t$ and let $e_{0}, \ldots, e_{q-2} \in E$ and $b \in \mathbf{B}_{j}$ be arbitrary. From (B2) it follows that there exists $a \in \mathbf{B}_{i}$ such that $a \stackrel{w}{\longrightarrow} b$. Since $\mathcal{B}_{w}(D)=E$, there exist $d_{0}, \ldots, d_{q-2}$ such that $d_{k} \stackrel{w}{\longrightarrow} e_{k}$ for all $k=0, \ldots, q-2$. It is not hard to see that $\stackrel{w}{\longrightarrow}$ is a subuniverse of $\mathbf{B}_{i} \times \mathbf{B}_{j}$, and therefore $t^{\mathbf{B}_{i}}\left(d_{0}, \ldots, d_{q-2}, a\right) \stackrel{w}{\longrightarrow} t^{\mathbf{B}_{j}}\left(e_{0}, \ldots, e_{q-2}, b\right)$. Since $D$ absorbs $\mathbf{B}_{i}$ we get $t\left(d_{0}, \ldots, d_{q-2}, a\right) \in D$ and conclude that $t\left(e_{0}, \ldots, e_{q-2}, b\right) \in E$. The same argument can be applied with permuted variables which proves equation $(*)$. To see that $E$ is a subuniverse of $\mathbf{B}_{j}$ it suffices to repeat the same reasoning for every operation of the algebra $\mathbf{A}$ taken in place of $t$ (with all $e_{i}$ 's and $b$ in $E$ ).

Let $\mathcal{L} \subseteq \mathcal{K}$ be a maximal component of the preorder $\leq$ which is bigger than $\left(K_{0}, i_{0}\right)$. If $(D, i),\left(D^{\prime}, i\right) \in \mathcal{L}$, then $(D, i) \leq\left(D^{\prime}, i\right) \leq(D, i)$ and the property $(B 6)$ implies that $D=D^{\prime}$ which allows us to define

$$
C_{i}= \begin{cases}D & \text { if there exists (unique) } D \text { such that }(D, i) \in \mathcal{L} \\ B_{i} & \text { otherwise }\end{cases}
$$

and put $\mathcal{C}=\left\{C_{i}, C_{i, j}=B_{i, j} \cap\left(C_{i} \times C_{j}\right): i, j<n\right\}$. Property $(B 6)$ for $\mathcal{B}$ together with maximality of $\mathcal{L}$ implies $(B 2)$ for $\mathcal{C}$ and therefore $\mathcal{C}$ is a $(1,2)$-system and a proper subsystem of $\mathcal{B}$. Moreover, by Claim (1), each $C_{i}$ absorbs $\mathbf{B}_{i}$, thus, in particular, $\mathcal{C}$ is compatible with $\mathbf{A}$.

To finish the proof we have to show that $\mathcal{C}$ satisfies $(B 4 S)$. Let $a, b \in C_{i}, w$ be a pattern based at $i$ and let us assume that $a$ and $b$ are connected in $\llbracket w \rrbracket$ in the strategy $\mathcal{B}$. Since $\mathcal{C}$ is a $(1,2)$-system we can find, for any $k$, a realization of $w^{k}$ inside $\mathcal{C}$ starting at $a$. As $C_{i}$ is finite, there exists $a^{\prime} \in C_{i}$ and natural numbers $k, l$ such that $a \stackrel{w^{k}}{\longrightarrow} a^{\prime}$ and $a^{\prime} \stackrel{w^{l}}{\longrightarrow} a^{\prime}$ in $\mathcal{C}$. Similarly (going backwards from $b$ ) we can find $b^{\prime}$ such that $b^{\prime} \stackrel{w^{k^{\prime}}}{\longrightarrow} b$ and $b^{\prime} \stackrel{w^{l^{\prime}}}{\longrightarrow} b^{\prime}$ also in $\mathcal{C}$. We assume that $l=l^{\prime}$ since we can, wlog, replace both numbers by $l l^{\prime}$. All the realizations up to now lie inside $\mathcal{C}$. Using the property $(B 5)$ of the strategy $\mathcal{B}$ we know that $a^{\prime} \stackrel{w^{l^{\prime \prime}}}{\longrightarrow} b^{\prime}$ for all big enough $l^{\prime \prime}$ (this realization needn't be in $\mathcal{C}$ ) and we assume, wlog, $l=l^{\prime \prime}$ (we can again replace $l, l^{\prime}$ and $l^{\prime \prime}$ by $l l^{\prime \prime}$ ).

Now take any $m$ smaller than the arity of $t$ and form the following matrix (with $\operatorname{ar}(t)$ rows): To the first $m$ rows write a realization of $w^{l}$ joining $a^{\prime}$ to $a^{\prime}$ inside $\mathcal{C}$, to the $(m+1)$-st row write a realization of $w^{l}$ joining $a^{\prime}$ to $b^{\prime}$, to the remaining rows write a realization of $w^{l}$ joining $b^{\prime}$ to $b^{\prime}$. By applying $t$ to columns, we obtain a realization of $w^{l}$ joining $t\left(a^{\prime}, \ldots, a^{\prime}, a^{\prime}, b^{\prime}, \ldots, b^{\prime}\right)$ (the letter $a^{\prime}$ appears $(m+1)$-many times) to $t\left(a^{\prime}, \ldots, a^{\prime}, b^{\prime}, b^{\prime}, \ldots, b^{\prime}\right)$. From the absorbing property of $t$ it follows that this realization lies in $\mathcal{C}$. Now $a \stackrel{w^{k}}{\longrightarrow} a^{\prime}=t\left(a^{\prime}, \ldots, a^{\prime}\right) \stackrel{w^{l}}{\longrightarrow} t\left(a^{\prime}, \ldots, a^{\prime}, b^{\prime}\right) \ldots \stackrel{w^{l}}{\longrightarrow}$ $t\left(b^{\prime}, \ldots, b^{\prime}\right)=b^{\prime} \stackrel{w^{k^{\prime}}}{\longrightarrow} b$ and this realization of a power of $w$ lies in $\mathcal{C}$ which finishes the proof. 


\subsection{The remaining case}

In this section we prove that a Prague strategy compatible with an $S D(\wedge)$ algebra has a solution, by showing that every such strategy, which is not a solution itself, contains a strong, proper substrategy compatible with the same algebra.

Let $\mathcal{B}$ be an arbitrary Prague strategy (of size $n$ ) compatible with an $S D(\wedge)$ algebra $\mathbf{A}$. If $\mathcal{B}$ is a solution there is nothing to prove. If, on the other hand, one of $\mathbf{B}_{i}$ 's contains a proper absorbing subuniverse then Theorem 4.12 provides a strong, proper substrategy compatible with $\mathbf{A}$.

The remaining case is when no $\mathbf{B}_{i}$ has a proper absorbing set and, wlog, $\left|B_{0}\right|>1$. Let $\theta$ be a maximal congruence of $\mathbf{B}_{0}$, let $\mathbf{B}_{0}^{\prime}$ denote the simple factor algebra $\mathbf{B}_{0} / \theta$ and let $C_{0}^{0}, \ldots, C_{0}^{m-1}$ enumerate the partition of $B_{0}$ into the equivalence classes of $\theta$.

We split the indexing set of $\mathcal{B}$ into two disjoint parts:

$$
\mathcal{Z}=\left\{i<n: \mathcal{B}_{0, i}\left(C_{0}^{0}\right)=B_{i}\right\}, \text { and } \mathcal{Y}=\{0,1, \ldots, n-1\} \backslash \mathcal{Z},
$$

and let

$$
C_{i}^{j}=\mathcal{B}_{0, i}\left(C_{0}^{j}\right), \quad j<m, i \in \mathcal{Y} .
$$

We define an algebra $\mathbf{M}$ to be an isomorphic copy of $\mathbf{B}_{0}^{\prime}$ with the universe $\{0,1, \ldots, m-1\}$ and with elements $C_{0}^{i}$ renamed to $i$. Thus for every $l$-ary term operation $t$ of $\mathbf{A}$ and any $k_{0}, \ldots, k_{l-1}<m$ we have

$$
t^{\mathbf{B}_{0}^{\prime}}\left(C_{0}^{k_{0}}, \ldots, C_{0}^{k_{l-1}}\right) \subseteq C_{0}^{t^{\mathrm{M}}\left(k_{0}, \ldots, k_{l-1}\right)} .
$$

From the fact that $\mathbf{B}_{0, i}$ is a subalgebra of $\mathbf{B}_{0} \times \mathbf{B}_{i}$ it follows that for all $i<n$

$$
t^{\mathbf{B}_{i}}\left(C_{i}^{k_{0}}, \ldots, C_{i}^{k_{l-1}}\right) \subseteq C_{i}^{t^{\mathrm{M}}\left(k_{0}, \ldots, k_{l-1}\right)} .
$$

The following three claims uncover a structure of the strategy $\mathcal{B}$.

Claim (1). For all $i \in \mathcal{Y}, B_{i}$ is a disjoint union of $C_{i}^{0} \ldots C_{i}^{m-1}$.

Proof. Let

$$
B_{0, i}^{\prime}=\left\{\left([a]_{\theta}, b\right):(a, b) \in \mathbf{B}_{0, i}\right\} .
$$

Then $B_{0, i}^{\prime}$ is a subdirect subuniverse of $\mathbf{B}_{0}^{\prime} \times \mathbf{B}_{i}$.

If $B_{0, i}^{\prime}$ is left separated, then the claim follows. Otherwise, by Lemma $4.9, B_{0, i}^{\prime}$ is fully linked. As $i \in \mathcal{Y}, \mathcal{B}_{0, i}\left(C_{0}^{0}\right) \varsubsetneqq B_{i}$ and therefore $B_{0, i}^{\prime}$ is a proper subuniverse of $\mathbf{B}_{0}^{\prime} \times \mathbf{B}_{i}$. Thus, by Theorem 4.11, either $\mathbf{B}_{0}^{\prime}$ has a proper absorbing set (and then its union is a proper absorbing set of $\mathbf{B}_{0}$ ) or $\mathbf{B}_{i}$ has a proper absorbing set, which, in both cases, is a contradiction.

Claim (2). For all $i, j \in \mathcal{Y}$ and all $k<m, \mathcal{B}_{i, j}\left(C_{i}^{k}\right)=C_{j}^{k}$.

Proof. From Claim (1) it follows that $\mathcal{B}_{(i, 0, j, 0, i)}\left(C_{i}^{k}\right)=C_{i}^{k}$. The property (B6) of $\mathcal{B}$ gives us $\mathcal{B}_{i, j}\left(C_{i}^{k}\right)=\mathcal{B}_{(i, 0, j)}\left(C_{i}^{k}\right)$, and the right side is, by Claim (1) again, equal to $C_{j}^{k}$.

Claim (3). For all $i \in \mathcal{Y}, j \in \mathcal{Z}$ and $k<m, \mathcal{B}_{i, j}\left(C_{i}^{k}\right)=B_{j}$.

Proof. Let $w=(0, i, j)$ and let $R \subseteq B_{0}^{\prime} \times B_{j}$ be the following subset

$$
R=\left\{\left([a]_{\theta}, b\right): a \stackrel{w}{\longrightarrow} b\right\}
$$

Clearly $R$ is a subdirect subuniverse of $\mathbf{B}_{0}^{\prime} \times \mathbf{B}_{j}$ and if $R=B_{0}^{\prime} \times B_{j}$, we get $B_{j}=\mathcal{B}_{w}\left(C_{0}^{k}\right)=$ $\mathcal{B}_{i, j}\left(C_{i}^{k}\right)$. It remains to exclude other options. If $R \neq B_{0}^{\prime} \times B_{j}$ is not left separated, we can derive a contradiction using Lemma 4.9 and Theorem 4.11 as in the proof of Claim (1). If $R \neq B_{0}^{\prime} \times B_{j}$ is left separated, then $\mathcal{B}_{(0, i, j, i, 0)}\left(C_{0}^{k}\right)=C_{0}^{k}$, in particular $\mathcal{B}_{(0, i, j)}\left(C_{0}^{k}\right)$ is a proper subset of $B_{j}$. But $\mathcal{B}_{(0, i, j)}\left(C_{0}^{k}\right)=\mathcal{B}_{0, j}\left(C_{0}^{k}\right)=B_{j}$ by the property $(B 6)$, a contradiction. 
For a subset $X \subseteq\{0,1, \ldots, m-1\}$ which is not necessarily a subuniverse of $\mathbf{M}$, let

$$
B_{i}^{X}= \begin{cases}\bigcup_{j \in X} C_{i}^{j} & \text { if } i \in \mathcal{Y} \\ B_{i} & \text { if } i \in \mathcal{Z},\end{cases}
$$

$B_{i, j}^{X}=B_{i, j} \cap\left(B_{i}^{X} \times B_{j}^{X}\right)$ and finally let $\mathcal{B}^{X}$ denote the system of sets $\mathcal{B}^{X}=\left\{B_{i}^{X}, B_{i, j}^{X}: i, j<n\right\}$.

By Claims (2) and (3), if $X \neq \emptyset$, then $\mathcal{B}^{X}$ is a $(1,2)$-system. Let

$$
\mathcal{R}=\left\{X \subseteq\{0,1, \ldots, m-1\}: \mathcal{B}^{X} \text { is a strong substrategy of } \mathcal{B}\right\}
$$

and note that we do not require compatibility of $\mathcal{B}^{X}$ with $\mathbf{A}$ even for $X \in \mathcal{R}$. Obviously $\{0,1, \ldots, m-1\} \in \mathcal{R}$. If $\mathcal{R}$ contains a one-element set $\{x\}$ we are done - since $B_{i}^{\{x\}}$ is either $B_{i}$ or a congruence class of an idempotent algebra, then the strategy $\mathcal{B}^{\{x\}}$ is compatible with $\mathbf{A}$, which means that $\mathcal{B}^{\{x\}}$ is a proper strong substrategy of $\mathcal{B}$ compatible with $\mathbf{A}$.

By comparing these remarks and Theorem 4.10, we see that the only missing piece is the following claim.

Claim. (4) If $x \in X \in \mathcal{R}$, then for any WNU term operation $t$ of $\mathbf{A}$ we have $\left\{t^{\mathbf{M}}(x, x, \ldots, x, y)\right.$ : $y \in X\} \in \mathcal{R}$.

Proof. Let $x, X$ and $t$ be as in the statement of the claim, let $\circ_{t}$ denote the binary operation $a \circ_{t} b=t(a, a, \ldots, a, b)$ and $Y=x \circ_{t} X$. We have to check that $\mathcal{B}^{Y}$ satisfies the property $(B 4 S)$. Let $i<n, w$ be any pattern based at $i$ and let $a, b \in B_{i}^{Y}$ be elements connected in $\llbracket w \rrbracket$ in $\mathcal{B}$. If $\llbracket w \rrbracket \subseteq \mathcal{Y}$, then necessarily $a, b \in B_{i}^{\{y\}}$ for some $y \in Y$ and any realization of $w^{j}$ connecting $a$ to $b$ (in $\mathcal{B}$ ) lies also in $\mathcal{B}^{\{y\}}$ (see Claim (2)) and thus in $\mathcal{B}^{Y}$.

Now assume $\llbracket w \rrbracket \nsubseteq \mathcal{Y}$. From Claims (2) and (3) it follows that there exist $a^{\prime}, b^{\prime} \in B_{i}^{\{x\}}$ such that $a \stackrel{w}{\longrightarrow} a^{\prime}$ and $b^{\prime} \stackrel{w}{\longrightarrow} b$ in $\mathcal{B}^{Y}$. Similarly as in the proof of Theorem 4.12 we can find $a^{\prime \prime}, b^{\prime \prime} \in \mathcal{B}^{\{x\}}$ such that $a^{\prime} \stackrel{w^{j}}{\longrightarrow} a^{\prime \prime}, b^{\prime \prime} \stackrel{w^{j^{\prime}}}{\longrightarrow} b^{\prime}$ in $\mathcal{B}^{\{x\}}$ and $a^{\prime \prime} \stackrel{w^{l}}{\longrightarrow} a^{\prime \prime}$ and $b^{\prime \prime} \stackrel{w^{l}}{\longrightarrow} b^{\prime \prime}$. Because $\mathcal{B}^{X}$ is a strong substrategy of $\mathcal{B}$, there exists a realization $a^{\prime \prime} \stackrel{w^{l^{\prime \prime}}}{\longrightarrow} b^{\prime \prime}$ in $\mathcal{B}^{X}$ for all sufficiently big $l^{\prime \prime}$ and, as before, we can assume $l=l^{\prime \prime}$.

As in the proof of Theorem 4.12 for every $j$ (smaller than the arity of $t$ ) we form the following matrix: To the first $j$ rows write a realization of $a^{\prime \prime} \stackrel{w^{l}}{\longrightarrow} a^{\prime \prime}$ in $\mathcal{B}^{\{x\}}$, to the $(j+1)$-st row write a realization of $a^{\prime \prime} \stackrel{w^{l}}{\longrightarrow} b^{\prime \prime}$ in $\mathcal{B}^{X}$ and to the remaining rows write a realization of $b^{\prime \prime} \stackrel{w^{l}}{\longrightarrow} b^{\prime \prime}$ in $\mathcal{B}^{\{x\}}$. When we apply the term $t$ to the columns of this matrix, we get a realization of $t\left(a^{\prime \prime}, \ldots, a^{\prime \prime}, a^{\prime \prime}, b^{\prime \prime}, \ldots, b^{\prime \prime}\right) \stackrel{w^{l}}{\longrightarrow} t\left(a^{\prime \prime}, \ldots, a^{\prime \prime}, b^{\prime \prime}, b^{\prime \prime}, \ldots, b^{\prime \prime}\right)$ in $\mathcal{B}^{x \circ_{t} X}=\mathcal{B}^{Y}$. Composition of our realizations gives us a realization of a power of the pattern $w$ connecting $a$ to $b$ in $\mathcal{B}^{Y}$ which finishes the proof.

\section{References}

[1] Libor Barto and Marcin Kozik. Congruence distributivity implies bounded width. manuscript, 2008.

[2] Libor Barto, Marcin Kozik, and Todd Niven. Graphs, polymorphisms and the complexity of homomorphism problems. In STOC '08: Proceedings of the 40th annual ACM symposium on Theory of computing, pages 789-796, New York, NY, USA, 2008. ACM. 
[3] Libor Barto, Marcin Kozik, and Todd Niven. The CSP dichotomy holds for digraphs with no sources and no sinks (a positive answer to a conjecture of Bang-Jensen and Hell). SIAM J. Comput., 38(5):1782-1802, 2009.

[4] Joel Berman, Paweł Idziak, Petar Marković, Ralph McKenzie, Matthew Valeriote, and Ross Willard. Varieties with few subalgebras of powers. Trans. Amer. Math. Soc. (to appear), 2006.

[5] Andrei Bulatov and Víctor Dalmau. A simple algorithm for Mal'tsev constraints. SIAM J. Comput., 36(1):16-27 (electronic), 2006.

[6] Andrei Bulatov, Peter Jeavons, and Andrei Krokhin. Classifying the complexity of constraints using finite algebras. SIAM J. Comput., 34(3):720-742 (electronic), 2005.

[7] Andrei A. Bulatov. Tractable conservative constraint satisfaction problems. In LICS '03: Proceedings of the 18th Annual IEEE Symposium on Logic in Computer Science, page 321, Washington, DC, USA, 2003. IEEE Computer Society.

[8] Andrei A. Bulatov. Combinatorial problems raised from 2-semilattices. J. Algebra, 298(2):321$339,2006$.

[9] Andrei A. Bulatov. A dichotomy theorem for constraint satisfaction problems on a 3-element set. J. ACM, 53(1):66-120 (electronic), 2006.

[10] Andrei A. Bulatov, Andrei A. Krokhin, and Peter Jeavons. Constraint satisfaction problems and finite algebras. In Automata, languages and programming (Geneva, 2000), volume 1853 of Lecture Notes in Comput. Sci., pages 272-282. Springer, Berlin, 2000.

[11] Andrei A. Bulatov, Andrei A. Krokhin, and Benoit Larose. Dualities for constraint satisfaction problems (a survey paper). LNCS 5250 (to appear), 2008.

[12] Catarina Carvalho, Víctor Dalmau, Petar Marković, and Miklós Maróti. CD(4) has bounded width. to appear in Algebra Universalis, 2007.

[13] Tomás Feder and Moshe Y. Vardi. The computational structure of monotone monadic SNP and constraint satisfaction: a study through Datalog and group theory. SIAM J. Comput., 28(1):57-104 (electronic), 1999.

[14] Peter Jeavons, David Cohen, and Marc Gyssens. Closure properties of constraints. J. ACM, 44(4):527-548, 1997.

[15] Emil Kiss and Matthew Valeriote. On tractability and congruence distributivity. Log. Methods Comput. Sci., 3(2):2:6, 20 pp. (electronic), 2007.

[16] Benoit Larose and László Zádori. Bounded width problems and algebras. Algebra Universalis, 56(3-4):439-466, 2007.

[17] Miklós Maróti and Ralph McKenzie. Existence theorems for weakly symmetric operations. Algebra Universalis, 59(3-4):463-489, 2008. 
Appendix 


\section{Proofs for Section "Reduction to Prague strategies"}

First we introduce an auxiliary notion of a $(2,3)$-system:

Definition 5.1. $A(1,2)$-system $\mathcal{B}=\left\{B_{i}, B_{i, j}: 0 \leq i, j<n\right\}$ is called a (2,3)-system, if for all $i, j, k<n$,

(B3) if $(a, b) \in B_{i, j}$ then there exists $c \in B_{k}$ such that $(a, c) \in B_{i, k}$ and $(b, c) \in B_{j, k}$.

Lemma 5.3 below proves that every $(2,3)$-system is a Prague strategy. This will fill the gap in the proof of Theorem 3.7, since from the properties $(S 1)-(S 3)$ of $\mathcal{F}$ it easily follows that $\mathcal{B}$ (constructed in the proof of Theorem 3.7 on page 6$)$ is a $(2,3)$-system. For a more detailed description of the reduction we refer to [1].

Lemma 5.2. Let $\mathcal{B}$ be $a(2,3)$-system of size $n$, let $i, j<n$ and let $w=\left(w_{0}, \ldots, w_{l}\right)$ be a pattern. Then $a \stackrel{w}{\longrightarrow} b$ for any $a \in B_{w_{0}}, b \in B_{w_{l}}$ such that $(a, b) \in B_{w_{0}, w_{l}}$.

Proof. Using (B3) from the definition of $(2,3)$-system to $(a, b)$ and the coordinates $i, j, w_{1}$ we obtain $c_{0} \in B_{w_{1}}$ such that $\left(a, c_{0}\right) \in B_{w_{0}, w_{1}}$ and $\left(c_{0}, b\right) \in B_{w_{1}, w_{l}}$. The element $c_{0}$ is the second (after $a$ ) element of a realization of the pattern $w$. Continuing the reasoning we use $(B 3)$ to $\left(c_{0}, b\right) \in B_{w_{1}, j}$ and the coordinates $w_{1}, j, w_{2}$ to obtain $c_{2}$ - the third element of a realization of $w$. Repeated applications of this reasoning produce a realization of the pattern $w$ connecting $a$ to $b$.

Lemma 5.3. Every $(2,3)$-system is a Prague strategy.

Proof. Let $i<n$, let $w$ be a pattern and let $a, b \in B_{i}$ be connected in $\llbracket w \rrbracket$, i.e. there exists a pattern $v=\left(v_{0}, \ldots, v_{l}\right)$ such that $a \stackrel{v}{\rightarrow} b$ and $\llbracket v \rrbracket \subseteq \llbracket w \rrbracket$. Let $a=a_{0}, \ldots a_{l}=b$ be a realization of $v$. Since $v_{1}$ appears in $w$ there exists an initial part of $w$, say $w^{\prime}$, starting with $i$ and ending with $v_{1}$. Since $\left(a, a_{1}\right) \in B_{i, v_{1}}$ we use Lemma 5.2 to connect $a$ to $a_{1}$ via $w^{\prime}$. Since $v_{2}$ appears in $w$ there exists $w^{\prime \prime}$ such that $w^{\prime} w^{\prime \prime}$ is an initial part of $w^{2}$ and such that $w^{\prime \prime}$ ends in $v_{2}$. Since $\left(a_{1}, a_{2}\right) \in B_{v_{1}, v_{2}}$ we use Lemma 5.2 again to connect $a_{1}$ to $a_{2}$ via a pattern $v_{1} w^{\prime \prime}$. Now $a_{0}$ and $a_{2}$ are connected via pattern $w^{\prime} w^{\prime \prime}$. By continuing this reasoning we obtain the pattern $w^{k}$ (for some $k$ ) connecting $a$ to $b$.

This concludes the reduction of Theorem 3.7 to Theorem 4.5. Finally we present a proof of the last lemma of the subsection.

Lemma (4.7). Let $\mathcal{B}$ be $(1,2)$-system. TFAE:

$(B 4) \mathcal{B}$ is a Prague strategy;

(B5) for every number $i<n$ and every pattern $w$ based at $i$ in $\mathcal{B}$ if $a, b \in B_{i}$ are connected in $\llbracket w$, then there exists a natural number $m$ such that, for all $k>m, a \stackrel{w^{k}}{\longrightarrow} b$;

(B6) for every number $i<n$ and every pattern $w$ based at $i$ in $\mathcal{B}$ if $K_{0} \subseteq B_{i}$ and $\mathcal{B}_{w}\left(K_{0}\right)=K_{0}$ then for any initial segment $w^{\prime}$ of $w$ ending with $j \mathcal{B}_{w^{\prime}}\left(K_{0}\right)=\mathcal{B}_{i, j}\left(K_{0}\right)$.

Proof. For $(B 4) \Longrightarrow(B 5)$ it is clearly enough to prove the claim for $a=b$ (as $a$ is connected to itself in any non-empty set). To do so, we obtain (using $(B 4)$ ) a natural number $p$ such that $a \stackrel{w^{p}}{\longrightarrow} a$. Let $c$ be an element of $B_{w_{0}}$ such that $a \stackrel{w}{\longrightarrow} c \stackrel{w^{p-1}}{\longrightarrow} a$. We use the property $(B 4)$ for $a, c$ and the pattern $w^{p}$ to find a natural number $q$ such that $a \stackrel{w^{p q}}{\longrightarrow} c$. From $a \stackrel{w^{p}}{\longrightarrow} a$ and $a \stackrel{w^{p q+p-1}}{\longrightarrow} a$ (as $a \stackrel{w^{p q}}{\longrightarrow} c \stackrel{w^{p-1}}{\longrightarrow} a$ ) we get $a \stackrel{w^{x p+y(p q+p-1)}}{\longrightarrow} a$ for arbitrary $x, y$. Since $p$ and $p q+p-1$ are coprime, the claim follows. 
For $(B 5) \Longrightarrow(B 6)$ let $i, j$ and $w, w^{\prime}$ be as in the statement and let $w^{\prime \prime}$ be such that $w=w^{\prime} w^{\prime \prime}$. We put $K_{1}=\mathcal{B}_{w^{\prime}}\left(K_{0}\right)$ and since $\mathcal{B}_{w}\left(K_{0}\right)=K_{0}$ we get $K_{1}=\mathcal{B}_{w^{\prime \prime} w^{\prime}}\left(K_{1}\right)$. Then (B5) implies that $K_{1}$ consists of all the elements of $B_{j}$ connected to $K_{1}$ in $\llbracket w \rrbracket$. Therefore $\mathcal{B}_{i, j}\left(K_{0}\right) \subseteq K_{1}$. Similarly $\mathcal{B}_{j, i}\left(K_{1}\right) \subseteq K_{0}$ and since $\mathcal{B}$ is a $(1,2)$-system we obtain $\mathcal{B}_{i, j}\left(K_{0}\right)=K_{1}$ as required.

For $(B 6) \Longrightarrow(B 4)$ we set $a, b$ and $i, w$ as in the statement and consider the sequence $\mathcal{B}_{w}(\{a\}), \mathcal{B}_{w^{2}}(\{a\}), \ldots$ As $B_{i}$ is finite the sequence will start repeating and we obtain numbers $p, q>0$ such that $K_{0}=\mathcal{B}_{w^{p}}(\{a\})=\mathcal{B}_{w^{q}}\left(\mathcal{B}_{w^{p}}(\{a\})\right)$. An application of $(B 6)$ immediately implies that $\mathcal{B}_{w}\left(K_{0}\right)=K_{0}$ and repeated applications of $(B 6)$ for various cyclic shifts (as in the previous case) of $w$ show that every element of $B_{i}$ connected in $\llbracket w \rrbracket$ to some element of $K_{0}$ is in $K_{0}$. Thus $b \in K_{0}$ and the implication is proved.

\section{Proofs of the algebraic results}

We start with Lemma 4.9 .

Lemma (4.9). Let $\mathbf{R}$ be a subdirect product of $\mathbf{C}$ and $\mathbf{D}$. If $\mathbf{C}$ is simple and $R$ is not left separated, then $R$ is fully linked.

Proof. The relation "to be linked to" is readily seen to be a congruence of $\mathbf{C}$. Since $\mathbf{C}$ is simple, "to be linked to" is either the diagonal or the full equivalence. In the first case $R$ is left separated, in the latter one $R$ is fully linked.

Our next aim is to prove Theorem 4.10. We introduce further notation.

Definition 6.1. Let $t$ be an $n$-ary operation and $\sigma$ be a permutation on the set $\{0,1, \ldots, n-1\}$. Then $t^{\sigma}$ is an $n$-ary operation defined by

$$
t^{\sigma}\left(a_{0}, \ldots, a_{n-1}\right)=t\left(a_{\sigma(0)}, a_{\sigma(1)}, \ldots, a_{\sigma(n-1)}\right) .
$$

Definition 6.2. Let $t$ be a WNU on a set $A$. Then $\circ_{t}$ is a binary, idempotent operation defined by

$$
a \circ_{t} b=t(a, a, \ldots, a, b) .
$$

$A W N U$ operation $t$ is called special, if for all $a, b \in A$

$$
a \circ_{t}\left(a \circ_{t} b\right)=a \circ_{t} b .
$$

Definition 6.3. Let $s$ be an operation on $A$ of arity $m, t$ be an operation on $A$ of arity $n$. By $s \triangleleft t$ we mean the mn-ary operation

$$
s \triangleleft t\left(a_{0}, \ldots, a_{m n-1}\right)=s\left(t\left(a_{0}, \ldots, a_{n-1}\right), t\left(a_{n}, a_{n+1}, \ldots\right), \ldots, t\left(\ldots, a_{m n-1}\right)\right) .
$$

For a natural number $k>0$ and an operation $t$, we put

$$
t^{\triangleleft k}=\underbrace{t \triangleleft t \triangleleft \cdots \triangleleft t}_{k \times} .
$$

The next lemma was proved in [17].

Lemma 6.4. For any $W N U$ operation $t$ on a set $A$, the operation $t^{\triangleleft|A| !}$ is a special $W N U$. 
Proof. For $a \in A$ we define a mapping $A \rightarrow A$ by $f_{a}(b)=a \circ_{t} b$. It is easily seen that $t^{\triangleleft i}$ is a WNU and that $a \circ_{t^{\triangleleft i}} b=f_{a}^{i}(b)$ for any $i$. Observe that $\left(f^{|A| !}\right)^{2}=f^{|A| !}$ for any mapping $f: A \rightarrow A$. This fact used for $f=f_{a}$ gives the desired conclusion.

One more lemma is necessary for proving the first theorem.

Lemma 6.5. Let $\mathbf{A}$ be a finite $S D(\wedge)$ algebra. Let

$$
\mathcal{K}=\left\{\circ: \exists k \forall l \geq k \text { there exists a WNU } t \in \text { Clo } \mathbf{A} \text { of arity l such that } \circ_{t}=\circ\right\} .
$$

Then

$(K 1) \mathcal{K} \neq \emptyset$.

(K2) If $\circ, \star \in \mathcal{K}$ then $\bullet \in \mathcal{K}$, where $a \bullet b=a \circ(a \star b)$.

(K3) If $\circ, \star \in \mathcal{K}$ then $\bullet \in \mathcal{K}$, where $a \bullet b=(a \star b) \circ a$.

Proof. (K1) Let $k=|A|$ ! and let $Z$ be an infinite set of natural numbers such that for all $z_{1}, z_{2} \in Z$, $z_{1} \neq z_{2}$, the numbers $z_{1}^{k}-1$ and $z_{2}^{k}-1$ are coprime. (Such a set can be constructed inductively: when we have a finite $Z$ satisfying the condition, then we can add one more element, namely $\prod_{z \in Z}\left(z^{k}-1\right)$.)

Since $\mathbf{A}$ has WNU term operations of all but finitely many arities, there exist distinct $z_{1}, z_{2} \in$ $Z$ and WNU term operations $t_{1}, t_{2}$ of arities $z_{1}, z_{2}$ (respectively) such that $\circ_{t_{1}}=\circ_{t_{2}}$.

Observe that given two special WNU operations $r, s$ of arities $i, j$ with $\circ_{r}=\circ_{s}$, we can form a WNU operation $v$ of arity $i+j-1$ such that $o_{v}=o_{r}$ by putting

$$
v\left(x_{0}, \ldots, x_{i+j-2}\right)=r\left(s\left(x_{0}, \ldots, x_{j-1}\right), x_{j}, \ldots, x_{i+j-2}\right) .
$$

By iterative application of this corollary it follows that from the special WNU term operations $v_{1}=t_{1}^{\triangleleft|A| !}$ and $v_{2}=t_{2}^{\triangleleft|A| !}$ we can form, for arbitrary natural numbers $i, j$, a WNU term operation $v$ of arity $i\left(z_{1}^{k}-1\right)+j\left(z_{2}^{k}-1\right)+1$ satisfying $\circ_{v}=\circ_{v_{1}}$. Since $z_{1}^{k}-1$ and $z_{2}^{k}-1$ are coprime, the claim follows.

(K2) If $t$ is an $n$-ary WNU operation such that $\circ_{t}=\circ$ and $s, s^{\prime}$ are WNU operations of arities $k, k^{\prime}$ such that $\circ_{s}=\circ_{s^{\prime}}=\star$, then $\circ_{v}=\bullet$, where

$$
v\left(x_{0}, \ldots, x_{(n-1) * k+k^{\prime}-1}\right)=t\left(s\left(x_{0}, \ldots, x_{k-1}\right), s\left(x_{k}, \ldots, x_{2 k-1}\right), \ldots, s^{\prime}\left(x_{(n-1) * k}, \ldots, x_{(n-1) * k+k^{\prime}-1}\right)\right)
$$

(K3) If $t$ is an $(n+1)$-ary WNU such that $\circ_{t}=\circ$ and $s$ is an $n$-ary WNU such that $\circ_{s}=\star$, then $o_{v}=\bullet$, where

$$
v\left(x_{0}, \ldots, x_{n}\right)=t\left(s\left(x_{1}, \ldots, x_{n}\right), s\left(x_{0}, x_{2}, \ldots, x_{n}\right), \ldots, s\left(x_{0}, \ldots, x_{n-1}\right)\right) .
$$

Theorem (4.10). Let $\mathbf{A}$ be a finite $S D(\wedge)$ algebra. Let $\mathcal{R}$ be a family of subsets of $A$ satisfying the following conditions

- $A \in \mathcal{R}$

- If $t \in$ Clo $\mathbf{A}$ is a WNU operation, $X \in \mathcal{R}$ and $x \in X$, then $x \circ_{t} X=\left\{x \circ_{t} y: y \in X\right\} \in \mathcal{R}$ 
Then $\mathcal{R}$ contains a one-element set.

Proof. Let $\mathcal{K}$ be as in the last lemma and take any WNU operation $t^{\prime} \in$ Clo $\mathbf{A}$ such that $\circ_{t^{\prime}} \in \mathcal{K}$. From (K2) it follows that there exists a WNU operation $t \in$ Clo $\mathbf{A}$ such that $o_{t} \in \mathcal{K}$ and

$$
a \circ_{t} b=\underbrace{a \circ_{t^{\prime}}\left(a \circ_{t^{\prime}}\left(\ldots \circ_{t^{\prime}}\left(a \circ_{t^{\prime}}\left(a \circ_{t^{\prime}} b\right)\right) \ldots\right)\right.}_{|A| !} .
$$

From the argument in Lemma 6.4 it follows that $t$ is a special WNU. For brevity we denote $\circ=o_{t}$.

Let $X$ be a member of $\mathcal{R}$ with minimal number of elements. Suppose, for a contradiction, that $|X|>1$. Therefore there exist $a_{1}, a_{2}^{\prime} \in X$ such that $a_{1} \neq a_{2}^{\prime}$. As $X$ is minimal, $\left|a_{1} \circ X\right|=|X|$, hence $a_{1}=a_{1} \circ a_{1} \neq a_{1} \circ a_{2}^{\prime}$. Let $a_{2}=a_{1} \circ a_{2}^{\prime}, X_{1}=a_{1} \circ X$. We have $a_{1} \neq a_{2}, a_{1}, a_{2} \in X_{1}$ and $a_{1} \circ a_{2}=a_{2}\left(\right.$ since $\left.a_{1} \circ a_{2}=a_{1} \circ\left(a_{1} \circ a_{2}^{\prime}\right)=a_{1} \circ a_{2}^{\prime}=a_{2}\right)$.

Let $a_{3}=a_{2} \circ a_{1}, X_{2}=a_{2} \circ X_{1} \in \mathcal{R}, a_{4}=a_{3} \circ a_{2}, X_{3}=a_{3} \circ X_{2} \in \mathcal{R}, \ldots$ Obviously $X_{i} \in \mathcal{R}$ and $a_{i}, a_{i+1} \in X_{i}$. Since $\left|X_{i}\right|=\left|X_{i-1}\right|$, it follows that $a_{i} \neq a_{i+1}$.

Let us define operations $\circ_{i}$ as follows:

$$
\begin{gathered}
\circ_{0}=\circ \\
a \circ_{i+1} b=(a \circ b) \circ_{i} a .
\end{gathered}
$$

From Lemma 6.5 (K3) it follows that all these operations are in $\mathcal{K}$.

Claim. For all $k$ and all $j \geq 2, a_{j} \circ_{k} a_{j-1}=a_{j+k+1}$

Proof. Since, for all $j \geq 2, a_{j} \circ a_{j-1}=a_{j+1}$ (from the definition of $a_{j+1}$ ), the claim holds for $k=0$. Assuming that the claim holds for $k$, the following calculation proves it for $k+1$ : for all $j \geq 2$

$$
a_{j} \circ_{k+1} a_{j-1}=\left(a_{j} \circ a_{j-1}\right) \circ_{k} a_{j}=a_{j+1} \circ_{k} a_{j}=a_{j+k+2} .
$$

Take arbitrary $n$ and $n^{\prime}$ so that $n<n^{\prime}$ and $a_{n}=a_{n^{\prime}}$. From the claim it follows that $a_{n}{ }^{\circ}\left(n^{\prime}-n-1\right)$ $a_{n-1}=a_{n^{\prime}}=a_{n}$. But then $a_{n}{ }^{\circ}{ }_{\left(n^{\prime}-n-1\right)} X_{n-1}$ has a smaller cardinality than $X_{n-1}$, because $X_{n-1}$ contains $a_{n-1}$ and $a_{n}$, a contradiction.

Now, in order to prove Theorem 4.11, we concentrate on a special case:

Lemma 6.6. Let $\mathbf{A}$ be a finite $S D(\wedge)$ algebra. Let $\mathbf{C}, \mathbf{D} \leq \mathbf{A}$ and $\mathbf{R} \leq \mathbf{C} \times \mathbf{D}$ be subdirect. Assume that there exists $d \in D$ such that $C \times\{d\} \subseteq R$. Then either $R=C \times D$ or there exists a proper absorbing set in $\mathbf{C}$ or $\mathbf{D}$.

Proof. We may assume that all operations of $\mathbf{A}$ are idempotent, otherwise we can replace $\mathbf{A}$ by the algebra of all idempotent term operations of $\mathbf{A}$.

Let $r, s \in$ Clo (A) be WNU operations such that

$$
a \circ_{s} b=\left(a \circ_{r} b\right) \circ_{r} a .
$$

Their existence is provided by Lemma 6.5.

Claim (1). If $X$ is a subuniverse of $\mathbf{C}$ such that $C \circ_{r} X=X$ and $C \circ_{s} X=X$, then $X$ absorbs $\mathbf{C}$ wrt. $\circ_{s}$.

Proof. For all $c \in C$ and $x \in X$ we have (trivially) $c \circ_{s} x \in X$ and $x \circ_{s} c \in X$ (as $x \circ_{s} c=\left(x \circ_{r} c\right) \circ_{r} x \in$ $X$ since $C \circ_{r} X=X$ ), thus $X$ absorbs $\mathbf{C}$ wrt. $\circ_{s}$. 
Assume $R \varsubsetneqq C \times D$. For any $X \subseteq C$ and $Y \subseteq D$ let

$$
\begin{aligned}
& X^{+}=\{d: \text { there exists } x \in X \text { such that }(x, d) \in R\} \\
& Y^{-}=\{c: \text { there exists } y \in Y \text { such that }(c, y) \in R\} .
\end{aligned}
$$

Note that if $X$ is a subuniverse of $\mathbf{C}$, then $X^{+}$is a subuniverse of $\mathbf{D}$.

For a subset $X \subseteq C$ we put

$$
N_{X}=\cap_{x \in X}\{x\}^{+}
$$

The assumptions of the lemma guarantees $N_{C} \neq \emptyset$ and $R \varsubsetneqq C \times D$ implies $N_{C} \neq D$. For any $X$, $N_{X}$ is a subuniverse of $\mathbf{D}$ - each $\{x\}$ is a subuniverse (from idempotency), therefore each $\{x\}^{+}$is a subuniverse, and the intersection of subuniverses is a subuniverse.

Claim (2). $N_{X}=N_{\langle X\rangle}$, where $\langle X\rangle$ is the subuniverse of $\mathbf{C}$ generated by $X$.

Proof. It follows from the fact that $\{d\}^{-}$is a subuniverse of $\mathbf{C}$ for any $d \in N_{X}$.

Claim (3). For any $X, Y \subseteq C$, any $W N U$ term operation $t \in$ Clo $\mathbf{A}$ and any permutation $\sigma$ we have $t^{\sigma}\left(N_{X}, \ldots, N_{X}, N_{Y}\right) \subseteq N_{X \circ{ }_{w} Y}$.

Proof. It is an easy consequence of the definitions and the fact that $R$ is a subuniverse of $\mathbf{C} \times \mathbf{D}$.

For a subuniverse $X$ of $\mathbf{C}$, let $f_{r}(X)=\left\langle C \circ_{r} X\right\rangle$. Similarly $f_{s}(X)=\left\langle C \circ_{s} X\right\rangle$.

If $X=f_{r}(X)$ and $f_{s}(X)=X$ for a proper subuniverse of $X$, then by Claim (1) $X$ is a proper absorbing subset of $\mathbf{C}$ and we are done.

Otherwise for all $c \in C$ we have $\ldots f_{r}\left(f_{s}\left(f_{r}(\{c\})\right)\right)=C$ for a sufficiently long chain. By Claims (2) and (3), $r^{\sigma}\left(N_{C}, \ldots, N_{C}, N_{X}\right) \subseteq N_{f_{r}(X)}$ for all $X \subseteq C$ and any permutation $\sigma$ and similarly for the operation $s$. It follows that $t=\cdots \triangleleft r \triangleleft s \triangleleft r$ satisfies $t^{\sigma}\left(N_{C}, \ldots, N_{C}, N_{\{c\}}\right) \subseteq N_{\ldots\left(f_{r}\left(f_{s}\left(f_{r}(\{c\})\right)\right) \ldots\right)}$ for all $c \in C$ and any permutation $\sigma$. We get $t^{\sigma}\left(N_{C}, \ldots, N_{C}, N_{\{c\}}\right) \subseteq N_{C}$. As $\cup_{c \in C} N_{\{c\}}=D$, the set $N_{C}$ absorbs $\mathbf{D}$ wrt. $t$.

Finally we are ready to prove the last theorem.

Theorem (4.11). Let $\mathbf{A}$ be a finite $S D(\wedge)$ algebra. Let $\mathbf{C}, \mathbf{D} \leq \mathbf{A}$ and let $\mathbf{R} \leq \mathbf{C} \times \mathbf{D}$ be subdirect and fully linked. Then either $R=C \times D$ or there exists a proper absorbing set in $\mathbf{C}$ or $\mathbf{D}$.

Proof. Let us assume $R \neq C \times D$.

Claim. (1) We can assume $N_{c}^{-}=C$ for all $c \in C$ (the notation is as in the last lemma).

Proof. If the condition is not satisfied, then $R \circ R^{-1}$ is a proper subset of $C \times C$ and we can work with $R \circ R^{-1} \leq \mathbf{C} \times \mathbf{C}$ instead of $R$. If the condition is still not satisfied, we again replace $R$ with $R \circ R^{-1}$. This process stops, since $R \circ R^{-1} \circ R \circ \cdots \circ R^{-1}=C \times C$ as $R$ is fully linked.

First suppose that there exists $\emptyset \neq X \subsetneq C$ such that for all $t \in$ Clo $\mathbf{A}$ there exists a permutation $\sigma$ such that $t^{\sigma}(X, C, C, \ldots, C)=X$. Such a set $X$ is clearly a subuniverse of $\mathbf{C}$ and $C \circ_{r} X=C$ and $C \circ_{s} X=C$, thus $X$ absorbs $\mathbf{C}$ wrt. $\circ_{s}$ (see Claim (1) in the previous proof).

So we can suppose that for all $\emptyset \neq X \subsetneq C$ there exists $t_{X} \in$ Clo $(\mathbf{A})$ such that $t_{X}^{\sigma}(X, C, C, \ldots, C)$ for all permutations $\sigma$. Let $C=\left\{c_{1}, \ldots, c_{m}\right\}$. The term operation

$$
t_{1}=t_{\left\{c_{1}\right\}} \triangleleft t_{\left\{c_{2}\right\}} \triangleleft \cdots \triangleleft t_{\left\{c_{m}\right\}}
$$

has the property that for all one-element subsets $X \subseteq C$ and for all permutations $\sigma, t_{1}^{\sigma}(X, C, \ldots, C)$ is at least two-element. Similarly (by composing through all the two-element subsets) we form a 
term operation $t_{2}$ such that $t_{2}^{\sigma}(X, C, \ldots, C)$ is at least three-element for all two-element $X$, etc. Now $t=t_{m-1} \triangleleft \cdots \triangleleft t_{1}$ satisfies $t^{\sigma}\left(\left\{c_{i}\right\}, C, \ldots, C\right)=C$ for all $i$ and for all permutations $\sigma$.

A subset $X$ of $C$ is good, if $N_{X}^{-}=C$. If $C$ is good, since then $N_{C}$ is nonempty and by the last lemma there exists a proper absorbing set in $\mathbf{C}$ or $\mathbf{D}$. Therefore we assume that $C$ is not good. On the other hand, by Claim (1) we can assume that $\{c\}$ is good for all $c \in C$. Take a maximal good subset $X$ of $C$. Claim (2) from the last lemma implies that $X$ is a subuniverse of $C$. Since $X$ is good, $N_{X \cup\{c\}} \neq \emptyset$ for any $c \in C$.

If $X$ is an absorbing set of $\mathbf{C}$ we are done. Otherwise there exist a coordinate $i, c_{i} \in C$ and $c_{0}, \ldots, c_{i-1}, c_{i+1}, \ldots, c_{n-1} \in X$ (where $n$ is the arity of $t$ ) such that $t\left(c_{0}, \ldots, c_{n-1}\right)=c \notin X$, therefore clearly

$$
t\left(N_{X \cup\left\{c_{0}\right\}}, N_{X}, N_{X}, \ldots, N_{X}\right) \subseteq N_{X \cup\{c\}} .
$$

As $X$ is a subuniverse of $\mathbf{C}, c_{i} \notin X$. For simplicity let us assume $i=0$.

Let $c^{\prime} \in C$ be arbitrary. Using the property of $t$ we find $c_{1}, \ldots, c_{n-1} \in C$ such that $t\left(c_{0}, c_{1}, \ldots, c_{n-1}\right)=c^{\prime}$. Since $X$ is good, there exist $d_{0} \in N_{X \cup\left\{c_{0}\right\}}$ and $d_{1}, \ldots, d_{n-1} \in N_{X}$ such that $\left(c_{j}, d_{j}\right) \in R$ for all $j=0, \ldots, n-1$. Now $\left(t\left(c_{0}, \ldots, c_{n-1}\right), t\left(d_{0}, \ldots, d_{n-1}\right)\right) \in R$, thus $\left(c^{\prime}, t\left(d_{0}, \ldots, d_{n-1}\right)\right) \in R$. The element $t\left(d_{0}, \ldots, d_{n-1}\right)$ lies in $N_{X \cup\{c\}}$ (see the inclusion in the last paragraph). We have shown that $X \cup\{c\}$ is good, a contradiction with maximality of $X$. 